\title{
Implementation of Orthogonal Frequency Division Multiplexing for SDR using MATLAB
}

\author{
R. Prameela Devi \\ CVR College of Engineering/ECE Department, Hyderabad, India \\ Email: prameela.rr@gmail.com
}

\begin{abstract}
Wireless Communication system is the emerging field of research. Users need to do many changes in the hardware to obtain high data rate less latency for less cost. To avoid Inter Symbol Interference (ISI) in a single carrier system, delay time must be very less compared to the symbol period. The data rate is inversely proportional to the symbol period. The low data rate is, due to the long symbol period. System with long symbol period $h$ is not considered as an efficient communication system. The multi carrier system like Frequency Division Multiplexing (FDM) technique, the available bandwidth is used for multi carrier transmission by dividing the total bandwidth to sub bands. To obtain high data rates, the multiple carriers can be placed closely in the spectrum. But due to the less gap between multiple carriers in the spectrum, there is a chance of having Inter Carrier Interference. To avoid this interference guard bands need to be inserted which in turn results in low data rate. This paper focuses on the Orthogonal Frequency Division Multiplexing (OFDM) system implementation for testing purposes and its verification. Most of the stages in a communication system can be replaced by the software. For this process a new system called software defined radio came to exist. SDR is a radio which can be used for signal processing with minimum cost. The main goal is to create an OFDM signal using MATLAB for signal processing, synchronization, equalization, demodulation and detection. The paper also gives the design of an OFDM transmitter and receiver for Software Defined Radio.
\end{abstract}

Keywords: FDM, OFDM, ICI, Software Defined Radio and MATLAB.

\section{INTRODUCTION}

The requirement for high speed data transmission has increased due to the rapid growth in the area of digital communication. To keep up with demand, a new modulation technique such as Orthogonal Frequency Division Multiplexing (OFDM) is currently being implemented. Now-a-days all the processor's power, has increased where the capability of handling the speed has become big task. A new multiplexing technique i.e. OFDM has become more important as it can have high speed with low interference. By studying the multi carrier system OFDM in many books and journals, it is clear that there will be a very good impact of OFDM in future communication system.

The main problem found in any communication system for high datarate is Inter Symbol Interference (ISI)[1]. ISI means the interference when a transmission interferes with itself and at receiver side it becomes difficult to decode the transmission correctly. As the receiver receives the signal from many obstacles by taking many reflected paths, the received signal will have many delayed versions of the original signal along with the actual one. This effect is called multipath. These delayed copies may cause interference at the receiver called ISI.

Main objective of any multi carrier system is efficient usage of spectrum, power consumption, robustness of multipath propagation and implementation complexity. Every objective with one or other may conflict, so implementations or techniques are selected which offer the best possible trade off between them. The best means of reducing the gap between the performance and channel capacity is OFDM modulation which combats multipath fading.

In addition to error correction coding techniques like turbo, spherical and Low density parity check codes, advanced OFDM is the good choice against multipath[2]. Fading effect can also be reduced to a certain extent. And to improve the efficiency of the spectrum the best method is to increase the capacity of the channel, which can be easily done with the new multi carrier system, OFDM.

In OFDM method, many orthogonal signals, which are overlapping are transmitted. It divides the available spectrum bandwidth into sub-channels. The gap between the sub-carriers is minimal theoretically such that a channel bandwidth is utilized. The main reason to opt OFDM is, it can handle the effects caused due to the multipath fading. Multipath causes mainly two problems - frequency selective fading and inter symbol interference. The achieved flat narrow band overcomes the frequency fading and modulation at low symbol rate removes the ISI by making long symbols than the channel impulse response. Using better error correcting methods along with frequency and time interleaving, more robustness can be achieved against frequency selective fading. And by inserting the required amount of guard band between the symbols of an OFDM signal, the effects of interference can be reduced even more so that at receiver, equalizer can be removed.

As the system increases information rate, the time required for each transmission becomes less. As there is no change in the delay time caused by the multipath, ISI is the main problem in a high speed communication. The new modulation method that is OFDM can avoid this problem by transmitting many low speed signals in parallel. Observe the below Figure.1 which shows two ways of transmitting the same data. If this transmission takes four seconds then each data will have duration of one second. But OFDM can send all the data in the same period simultaneously.

A Software Defined Radio (SDR) is a system where it uses software methods on digital signal. In a communication system after doing hardware related processing, the signal is converted into digital form as and then it is sent as input 
to the software system. The main aim of SDR is "to turn hardware problems into software problems" [3], so that radio engineers can handle the problems in more convenient domain. But everything cannot be converted into software, so SDR has two main sections: Software defined sub-system and Hardware defined sub-system. But this separation is not fixed. In general the antenna and the Analog to Digital Convertor (ADC) are of hardware defined and remaining system is of software. For this SDR we need software which can easily change the parameters like modulation type, signal to noise ratio, noise level, signal strength etc. which can be adapted to any other type of communication. That is hardware problems are converted to software problems.

\section{ORTHOGONAL FREQUENCY DIVISION MULTIPLEXING}

Mathematical Description

The OFDM Signal is given as

$\nu(t)=\sum_{k=0}^{N-1} X_{k} e^{j 2 \pi k t / T}, \quad 0 \leq t<T$,

where $\mathrm{Xk}$ is the number of symbols, the number of sub carriers is denoted by $\mathrm{N}$ and $\mathrm{T}$ is the total symbol time of OFDM.The spacing of $1 / \mathrm{T}$ makes the OFDM signal orthogonal for every symbol .

$$
\begin{aligned}
& \frac{1}{T} \int_{0}^{T}\left(e^{j 2 \pi k_{1} t / T}\right)^{*}\left(e^{j 2 \pi k_{2} t / T}\right) d t \\
= & \frac{1}{T} \int_{0}^{T} e^{j 2 \pi\left(k_{2}-k_{1}\right) t / T} d t=\delta_{k_{1} k_{2}}
\end{aligned}
$$

To avoid interference in channels due to multipath fading guard interval is included during this period a cyclic prefix is inserted before the OFDM block. OFDM with cyclic prefix is given as

$$
\nu(t)=\sum_{k=0}^{N-1} X_{k} e^{j 2 \pi k t / T}, \quad-T_{\mathrm{g}} \leq t<T
$$

Finally the transmitted signal is represented as

$$
\begin{aligned}
s(t) & =\Re\left\{\nu(t) e^{j 2 \pi f_{c} t}\right\} \\
& =\sum_{k=0}^{N-1}\left|X_{k}\right| \cos \left(2 \pi\left[f_{c}+k / T\right] t+\arg \left[X_{k}\right]\right)
\end{aligned}
$$
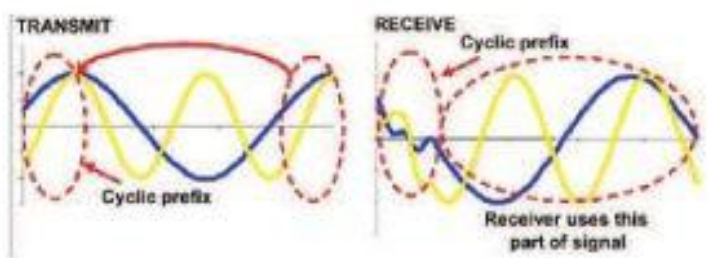

Figurel. Signal with cyclic prefix

\section{SOFTWARE DEFINED RADIO}

SDR is a radio platform which uses software methods on digital radio signals. That is at receiver the signal is digitized and given as input to the software domain. The main objective of SDR is to convert hardware related issues into software related issues so that the engineers can work in a software platform. In general, an SDR is divided into two parts: the hardware system and the software system.

For big requirements like designing and handling receiver with multiple FM channels at the same time, we need wide spectrum and powerful system to process all the data. The system based on OFDM has a wide band application and so it is more suitable system for SDR.

\section{ARChtrecture Of Orthogonal Frequency DIVISION MULTIPLEXING}

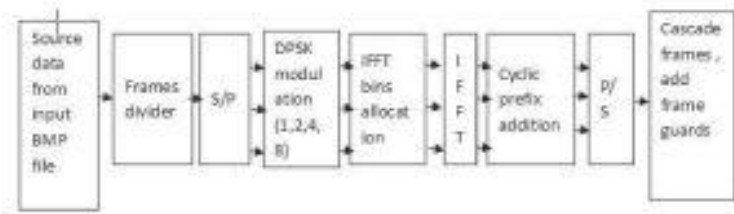

Figure 2. OFDM transmitter

\section{A. Frame Guard}

The main block in the OFDM transmitter is the modulator. Modulator modulates the input data. This modulated digital data stream is divided into frames based on variable symbol-per-frame, which gives the number of symbols per frame for a carrier.

Here in this paper the data is taken from an image file. Image pixel data is converted into matrix and then the matrix data is chunked based on the modulation type choosen. Here the number of symbols per frame is decided using modulation type If the number of symbols to be transmitted of a data stream, is less than a number of symbols per frame then the data is not converted into frames. And also if there is no data stream sufficiently long to divide it into multiple frames, then zeros are padded with two guard interval at either of the ends of the signal. This is used to find the starting point of substantial portion of the signal.

\section{B. OFDM Modulator}

The modulator generates a PSK signal matrix composed of complex numbers where the phases are the translated phases and all the magnitudes are considered as ones. For the remaining processing these complex numbers are converted into rectangular form[4]. 
Orthogonality between carriers must be maintained by controlling all the carriers and then OFDM process is done. OFDM signal is generated by taking input data like modulation type and signal power and then it chooses the spectrum based on its input. Each carrier to be produced is assigned some data to transmit. The required amplitude and phase of the carrier is then calculated based on the modulation type. The spectrum is then converted back to its time domain signal using an Inverse Fourier Transform.

Before differential or PSK modulation, encoding can be applied on each carrier (column of the matrix), and an extra row of reference data must be added to the matrix. The modulator then creates a row of random numbers within an interval defined by the symbol size (order of PSK chosen) and appends it on the top of the matrix. For every column, beginning from the second row (the first actual data symbol), the value is converted into the remainder of the sum of its previous row values and the value itself, over the size of the symbol (power 2 of the PSK order).

\section{IFFT-Spectral Space to Time Signal}

Each column of the DPSK matrix represents a carrier, their values are stored to the columns of the IFFT matrix at the locations where their corresponding carriers should reside. Their conjugate values are stored to the columns corresponding to the locations of the conjugate carriers. All other columns in the IFFT matrix are set to zero. To obtain the transmitting time signal matrix, Inverse Fast Fourier Transform (IFFT) of this matrix is taken. Only the real part of the IFFT result is useful, so the imaginary part is discarded,

\section{Cyclic prefix insertion}

A copy of the last $25 \%$ portion of every symbol period (Le. row of the matrix) is included in the beginning of a row. This is the periodic time guard which keeps the receiver to synchronize while demodulating each symbol period of the received signal [5]. The matrix is now a modulated matrix. The modulated matrix is converted into serial data and it is considered as a modulated signal for a frame.

\section{E. Communication Channel}

The channel simulation allows finding common wireless channel characteristics such as noise, multipath, and clipping. To the transmitted signal random data is added, which is considered as a simple noise and simulated. For multipath simulation, attenuated and delayed copies of the transmitted signal is added to the original signal which reflects the problem in wireless communication for multipath fading. After adding the attenuated and delayed signal, it is sent to the receiver and simulated.

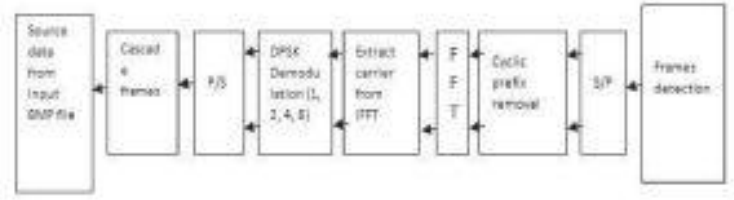

Figure 3, OFDM receiver

\section{F. Frame detector}

A trunk of received signal in a selective length is processed by the frame detector in order to determine the start of the signal frame. For only the first frame, this chosen part is comparatively large for taking the header into account [6]. The selected portion of received signal is sampled to a shorter discrete signal with a sampling rate defined by the system. A moving sum is taken over this sampled signal. The index of the minimum of the sampled signal is approximately the start of the frame guard while one symbol period farther from this index is the approximate location for the start of the useful signal frame [7].

The frame detector will then collect a moving sum of the input signal from about $10 \%$ of one symbol period before the start of the frame guard to about thirty percent of symbol period farther than the assumed start of the useful signal frame. The first portion, with a length of one less than a symbol period of this moving sum is discarded. The first minimum of this moving sum is the detected start of the useful signal frame.

\section{G. OFDM Demodulator}

Received OFDM signal is demodulated frame by frame. The total number of frames may vary by a wide range depending on the total amount of information transmitted through the OFDM System.

It is a reverse process of OFDM modulation. And like its modulator, the OFDM demodulator demodulates the received data frame by frame.

\section{H. Guard Time removal}

After converting a frame of discrete time signal from serial to parallel, a $25 \%$ length of a symbol period is removed from all rows. The remaining length of the symbol from all discrete signals is then lined up in parallel.

\section{FFT}

To the received signal is a Fast Fourier Transform (FFT) is applied which gives the spectrum of the received signal. The carriers are extracted to get the complex matrix of the received data.

\section{J. Phase Shift Keying (PSK) Demodulation}

The phase of each element in the matrix is changed and converted as a value within the size of the symbol.

On this new matrix differential operation is performed to get the demodulated data. This differential operation is used to calculate the difference between adjacent symbols in a column of the matrix. Then this parallel data is converted to serial and the demodulated stream of data for this frame is obtained. The series of zeros are appended to the data while transmitting to maintain the same symbol size for each carrier. With the help of number of symbols in a data and number of carriers the total number of appended zeros can be calculated. Similarly, for any type of PSK demodulation corresponding operation is performed to retrieve the original data. Here are the results shown for BPSK, QPSK, 16 PSK and 256PSK. 


\section{SOFTWARE. ALGORITHM}

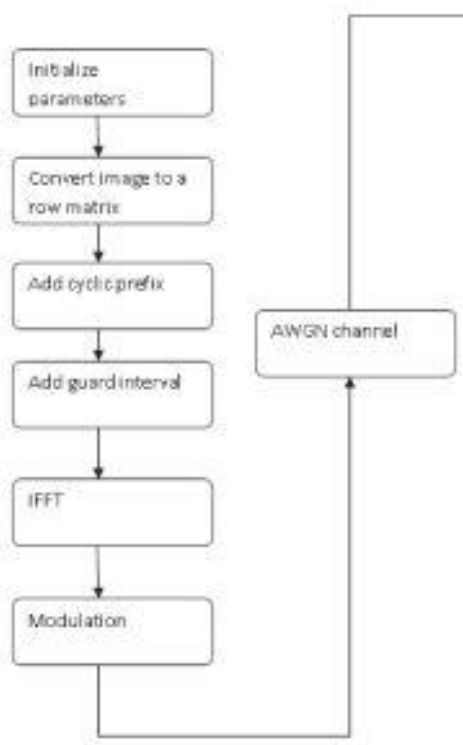

VI. RESULTS

A script file to invoke the OFDM parameters is simulated first, which invokes all the parameters of OFDM and initializes all the variables to start the simulation. Some variable values are allowed to the user to enter. The other variable values are obtained from the input variable data.

Please enter the data:

1) Image file name- an 8-bit grayscale (256 levels) bmp file;

2) IFFT length - (power of two);

3) total number of carriers - less than

[(IFFT size)/2-2];

4) Modulation type - PSK;

5) Clipping of peak power in $\mathrm{dB}$;

6) SNR in dB.

Following figures are the resultant images for different types of modulation methods.

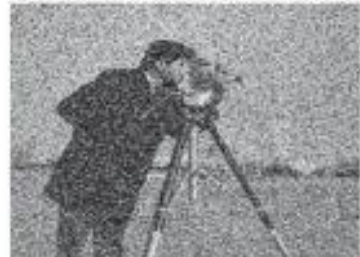

(a)

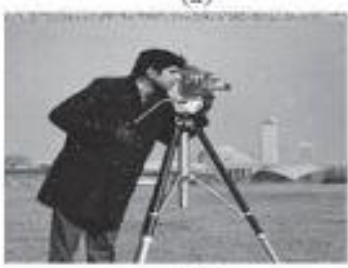

(c)

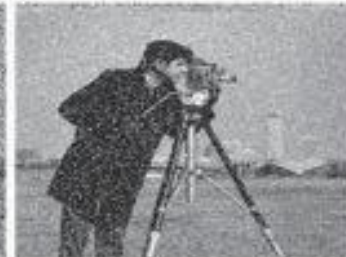

(b)

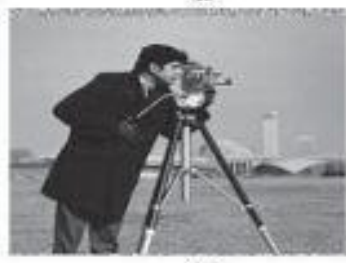

(d)
Figure 4: Images received at the OFDM receiver with BPSK technique for (a) $\mathrm{SNR}=0$ (b) $\mathrm{SNR}=5$ (c) $\mathrm{SNR}=10$ (d)SNR $=20$

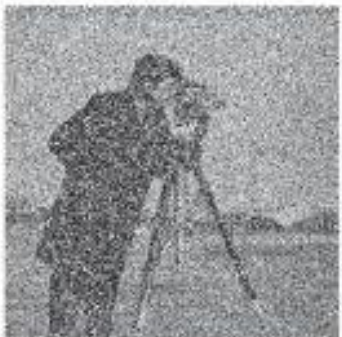

(a)

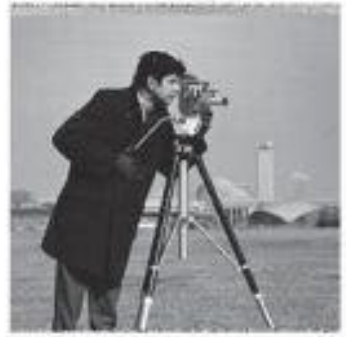

(c)

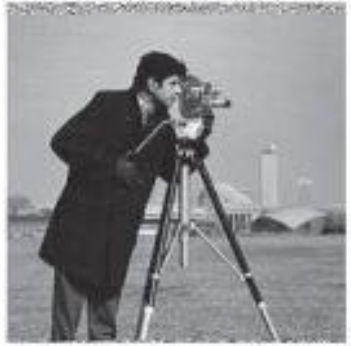

(e)

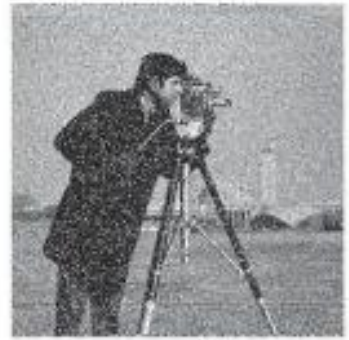

(b)

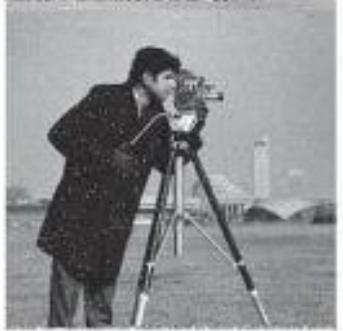

(d)

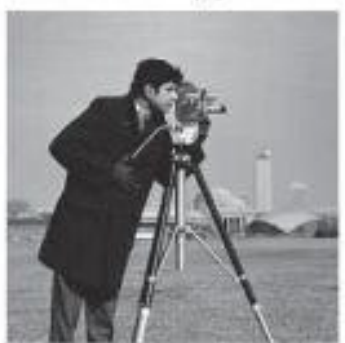

(f)
Figure 5: Images received at the OFDM receiver with QPSK technique for (a) $\mathrm{SNR}=0$ (b) $\mathrm{SNR}=5$ (c)SNR=10 (d)SNR=15 (e)SNR=20 (f)SNR=25

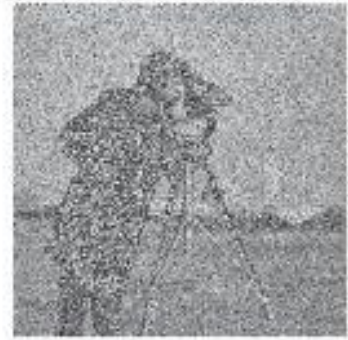

(a)

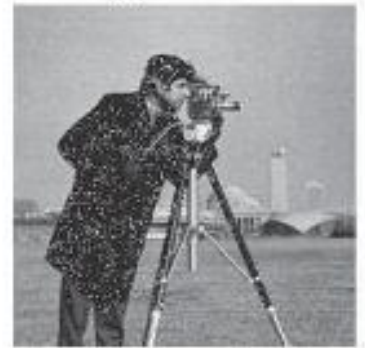

(c)

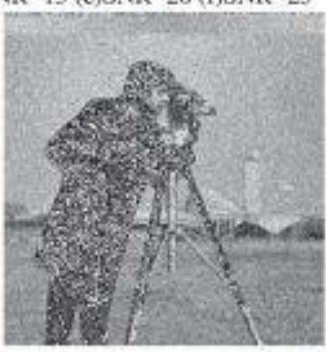

(b)

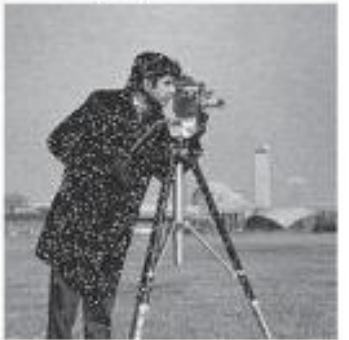

(d)
Figure 6: Images received at the OFDM receiver with 16PSK technique for (a) $S N R=0$ (b) $S N R=10$ (c)SNR=35 (d)SNR=40 


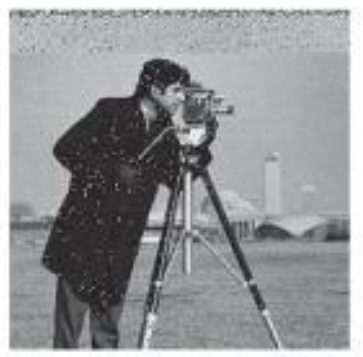

(a)

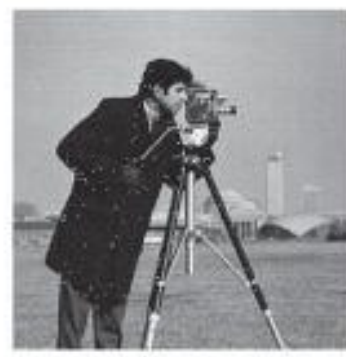

(b)
Figure 7: Images received at the OFDM receiver with 256 PSK technique for (a) SNR $=20$ (b) SNR $=50$

Table shows comparison of different parameters for PSK . QPSK, 16PSK and 256 PSK modulation techniques for the following input data. It is clearly observed that in any modulation technique as signal to noise ratio increases the error rate decreases. Transmission time and receiving time decreases as number of bits increased for PSK.

TABLE 1.

COMPARISON TABLE

\begin{tabular}{|c|c|c|c|c|}
\hline Modulation & SNR & BER $(\%)$ & $\begin{array}{c}\text { Average phase } \\
\text { error } \\
\text { (degree) }\end{array}$ & $\begin{array}{l}\text { \% pixel } \\
\text { error }\end{array}$ \\
\hline \multirow[t]{6}{*}{ BPSK } & 0 & 14.745 & 48.21 & 70,77 \\
\hline & 5 & 1.61 & 25.1 & 9.045 \\
\hline & 10 & 0.701 & 14.31 & 1.626 \\
\hline & 15 & 0.648 & 8.33 & 1.58 \\
\hline & 20 & 0.669 & 5.236 & 1.588 \\
\hline & 25 & 0.000 & 2.299 & 0.000 \\
\hline \multirow[t]{6}{*}{ QPSK } & 0 & 42.98 & 48.31 & 89.10 \\
\hline & 5 & 16.07 & 26.39 & 47.85 \\
\hline & 10 & 3.375 & 16.04 & 8.222 \\
\hline & 15 & 2.485 & 12.11 & 3.919 \\
\hline & 20 & 2.634 & 9.929 & 3.822 \\
\hline & 25 & 0.000381 & 7.196 & 0.0015 \\
\hline \multirow[t]{4}{*}{ 16PSK } & 0 & 83.99 & 48.609 & 97,41 \\
\hline & 5 & 71.71 & 27.39 & 91.91 \\
\hline & 10 & 53.87 & 17.37 & 78,00 \\
\hline & 35 & 17.00 & 6.53 & 31.04 \\
\hline \multirow[t]{2}{*}{ 256PSK } & 20 & 93.15 & 9.79 & 93.15 \\
\hline & 50 & 93.76 & 7.32 & 93.76 \\
\hline
\end{tabular}

Table 1. Shows comparison of different parameters for PSK, QPSK, 16PSK and 256 PSK modulation techniques for the given input data. It is clearly observed that in any modulation technique as signal to noise ratio increases the error rate decreases. And Transmission time and receiving time decreases as number of bits increased for PSK. As signal to noise ratio increases pixel error also decreases

\section{CONCLISIONS}

An OFDM modulation and demodulation is successfully simulated using MATLAB in this paper. All main components of an OFDM system are implemented. Some of the problems faced while developing the OFDM simulation program are, mapping the modulation and demodulation and also maintaining the data format throughout the process.

Possible future works are adding a feature to accept the data in word size rather in bits, and including many other modulation techniques other than PSK like QAM, implementing multiplexing for OFDM for multiple inputs; that is even increasing the data rate. With increase in data rate performance of the system may degrade which can be still enhanced by including the diversity methods. So to overcome this, Forward Error Correction can be implemented

\section{REFERENCFS}

[1] Usama S. Mohammed, H. A. Hamada, "Image transmission over OFDM channel with rate allocation scheme and minimum peak-1o average power ratio," Joumal of Telecommunications, Volume 2, Issue 2, May 2010.

[2] Pawan Sharma, Seema Verma, "Performance Analysis of Peak-to-Average Power Ratio Reduction Techniques for Wireless Communication Using OFDM Signals", IJCSI International Journal of Computer Science Issues, Vol. 7 , Issue 6, November 2010.

[3] Marek TICHY, Karel ULOVEC, "OFDM System Implementatiopn Using a USRP for Testing Puposes,"22nd International Conference Radioelektronika 2012.

[4] Roshan Jain, Sandhya sharma, "Simulation and performance analysis of OFDM with different modulation lechniques" International Joumal of Engineering and Technical Research (IJETR) ISSN: 2321-0869, Volume-1, Issue-1, March 2013.

[5] Wasiu Lawal" Adewuyi,s.O Ogunti,e.O, "Effect of Cyclic Prefix on OFDM over AWGN Channel," Intemational Journal of Innovative Research in Advanced Engineering (IJIRAE) ISSN: 2349-2163 Volume 1 Issue 9 (October 2014).

[6] Stefan H. Muller,Robert W. Bauml,Robert F. H. Fischer and Johannes B Huber, “OFDM with reduced Peak-to-Average Power Ratio by multiple signal representation". In Annals of Telecommunications, vol.52,no.1-2,pp. 58 67, February 1997.

[7] Joydev Ghosh, Abhishek Dey, Subham Bachhar, "Performance Analysis by Improving Bit Error Rate (BER) through various Diversity Techniques in Wireless Commanication, International Journal of Computer Applications (0975 - 8887) Volume 95- No. 9, June,2014 